\title{
LSS-GAC - A LAMOST Spectroscopic Survey of the Galactic Anti-center
}

\author{
X.-W. Liu ${ }^{1,2}$, H.-B. Yuan ${ }^{2}$, Z.-Y. Huo ${ }^{3}$, L.-C. Deng ${ }^{3}$, J.-L. Hou ${ }^{4}$, \\ Y.-H. Zhao ${ }^{3}$, G. Zhao ${ }^{3}$, J.-R. Shi ${ }^{3}$ and A.-L. Luo ${ }^{3}$, M.-S. Xiang ${ }^{1}$, \\ H.-H. Zhang ${ }^{1}$, Y. Huang ${ }^{1}$, H.-W. Zhang ${ }^{1} \dagger$ \\ ${ }^{1}$ Department of Astronomy, Peking University, Beijing, P. R. China \\ email: x.liu@pku.edu.cn \\ ${ }^{2}$ Kavli Institute for Astronomy and Astrophysics, Peking University, Beijing, P. R. China \\ ${ }^{3}$ National Astronomical Observatories, Chinese Academy of Sciences, Beijing, P. R. China \\ ${ }^{4}$ Shanghai Astronomical Observatory, Chinese Academy of Sciences, Shanghai, P. R. China
}

\begin{abstract}
As a major component of the LAMOST Galactic surveys, the LAMOST Spectroscopic Survey of the Galactic Anti-center (LSS-GAC) will survey a significant volume of the Galactic thin/thick disks and halo in a contiguous sky area of $\sim 3,400$ sq.deg., centered on the Galactic anti-center $\left(|b| \leqslant 30^{\circ}, 150 \leqslant l \leqslant 210^{\circ}\right)$, and obtain $\lambda \lambda 3800-9000$ low resolution $(R \sim 1,800)$ spectra for a statistically complete sample of $\gtrsim 3 \mathrm{M}$ stars of all colors, uniformly and randomly selected from $(r, g-r)$ and $(r, r-i)$ Hess diagrams obtained from a CCD imaging photometric survey of $\sim 5,400$ sq.deg. with the Xuyi 1.04/1.20 m Schmidt Telescope, ranging from $r=14.0$ to a limiting magnitude of $r=17.8$ (18.5 for limited fields). The survey will deliver spectral classification, radial velocity $\left(V_{\mathrm{r}}\right)$ and stellar parameters (effective temperature $\left(T_{\text {eff }}\right)$, surface gravity $(\log g)$ and metallicity $[\mathrm{Fe} / \mathrm{H}])$ for millions of Galactic stars. Together with Gaia which will provide accurate distances and tangential velocities for a billion stars, the LSS-GAC will yield a unique data set to study the stellar populations, chemical composition, kinematics and structure of the disks and their interface with the halo, identify streams of debris of tidally disrupted dwarf galaxies and clusters, probe the gravitational potential and dark matter distribution, map the 3D distribution of interstellar dust extinction, search for rare objects (e.g. extremely metal-poor or hyper-velocity stars), and ultimately advance our understanding of the assemblage of the Milky Way and other galaxies and the origin of regularity and diversity of their properties.
\end{abstract}

The survey was initiated in the fall of 2012 and expected to complete in the spring of 2017 . Hitherto, about $0.4 \mathrm{M}$ spectra of $\mathrm{S} / \mathrm{N}(\lambda 7450) \geqslant 10$ per pixel have been accumulated. In addition, bright nights have been used to target stars brighter than $14 \mathrm{mag}$ and have so far generated over $0.4 \mathrm{M}$ spectra, yielding an excellent sample of local stars to probe the solar neighborhood. LSP3, a set of pipelines tailored to the need of LSS-GAC, for spectral flux-calibration, and radial velocity and stellar parameter determinations, have been developed at Peking University, based on packages developed for the SDSS and those at the National Astronomical Observatories of Chinese Academy of Sciences. Comparisons of multi-epoch observations, with the SDSS results, as well as applying the pipelines to open and globular clusters show that LSP3 has achieved a precision of $5 \mathrm{~km} \mathrm{~s}^{-1}, 110 \mathrm{~K}, 0.15$ dex and 0.15 dex for $V_{\mathrm{r}}, T_{\text {eff }}, \log g$ and $[\mathrm{Fe} / \mathrm{H}]$, respectively. The data are publicly available, subject to regulations of the LAMOST data policy, and begin to yield scientific results. The potential of LSS-GAC and power of existing data are illustrated with examples of selected problems.

Keywords. The Milky Way, Surveys - photometry, Surveys - spectroscopy

$\dagger$ On behalf of the LAMOST Science Committee, LAMOST Scientific Performance Working Group and the LAMOST Galactic Surveys Working Group. 


\section{Scientific motivations}

Galaxies are building blocks of the universe. Understanding how galaxies assemble and acquire their characteristic structure and properties (the "grand designs") is one of the most challenging problems of astrophysics. In the current $\Lambda$ CDM paradigm, all cosmic structures including galaxies build up hierarchically by merging and accreting smaller ones. Although supported by extensive observations and large scale numerical simulations, the scenario involves many currently poorly understood and often highly non-linear baryonic physics (e.g. star formation and feedback) that it is compulsory to find out empirically the assemblage history of galaxies via detailed study of large samples of member stars of nearby, well-resolved galaxies, and inevitably less sophisticated statistical analysis of large samples of more distant galaxies (e.g. Mayer, Governato \& Kaufmann 2008). Both approaches depend on the acquisition of large survey samples.

The Milky Way is an archetypical disk galaxy and the only grand-design (barred) spiral that individual constituent stars can be resolved and studied multi-dimensionally (in 3D space position and velocity, and in chemical composition). On the other hand, owing to our own position inside the system, the roughly 200 billion Galactic stars are distributed over the whole sky of $4 \pi$ steradian, and their views are restricted by the ubiquitous intervening interstellar dust grains, in particular in the disk and towards the Galactic center. The study is further hampered by the difficulty of obtaining accurate distances to individual stars. Owing to the horrendous technical challenges of a complete spectroscopic census of the Galaxy, far beyond the capabilities of all existing facilities, hitherto progress of Galactic spectroscopic surveys lags far behind that of extragalactic, and as a consequence, our knowledge of our own host galaxy (contents of baryonic and dark matter, kinematics and chemical composition) is, ironically, not as complete and precise as that of the universe as a whole.

Modern large scale surveys, represented by the extremely successful SDSS (York et al. 2000), have revolutionized our understanding of galaxy formation and evolution, including the still on-going assemblage process of the Milky Way. On the other hand, being primarily an extragalactic program, the volume of the Galactic disk, in particular the thin disk, sampled by the SDSS, including its Galactic extensions SEGUE (Yanny et al. 2009) and APOGEE (Eisenstein et al. 2011; M. Schultheis, this volume), is very limited. Of the 0.7 M stellar spectra released in the SDSS DR9 (Ahn et al. 2012), a significant number were secured as the auxiliary data (e.g. as flux or radial velocity standard stars), with the remaining distributed in hundreds of disconnected fields, with targets selected from a host of algorithms. It is thus difficult to carry meaningful statistical analyses in terms of the underlying stellar populations. The operation of the Chinese LAMOST (Cui et al. 2012; Deng et al., this volume) opens up the possibility of carrying out a systematic spectroscopic survey that samples a significant volume of the Milky Way, in particular the disk, the defining structure that contains more than $90 \%$ of the baryonic matter and essentially all the angular momentum of the Galaxy. Compared to the facility of SDSS, the biggest strength of LAMOST is its sheer number of fibers $-4,000$, which is respectively a factor of 6.25 and 4 larger than that employed in the SDSS and SDSS-II, and in the SDSS-III. Most clear nights of the Xinglong Station where the LAMOST is located are distributed from September to March of the coming year, peaking in December when the Galactic anti-center $\left(\mathrm{RA}=5^{\mathrm{h}} 46^{\mathrm{m}}\right.$, Dec $\left.=+28^{\circ} 56^{\prime}\right)$ culminates around midnight. The outer disk also suffers less severe dust extinction. Most important of all, the outer parts of the Galactic disk have already revealed complex structure that is poorly understood. LSS-GAC, a LAMOST Spectroscopic Survey of the Galactic Anti-center and a major component of the LAMOST Experiment for Galactic Understanding and 
Exploration (LEGUE) proposed by the LAMOST Galactic Surveys Working Group, thus offers an unique opportunity to address questions key to revealing the true multidimensional structure and the formation and evolution history of the Galactic disk, and of the Galaxy as a whole. Examples of selected "puzzles" that can be tackled with the LSS-GAC include disk formation of the Milky Way and other late type spirals, secular evolution and (in)stability to gravitational perturbations of the disk, origin of the thick disk, structures and substructures of the outer disk [truncation, warps and flares, the Monoceros Ring and other anti-center stellar (sub)structures].

The LSS-GAC will form excellent synergy with Gaia, the forthcoming next generation space all-sky astrometric survey (Perryman et al. 2001; A. Vallenari, this volume). With an unprecedented astrometric accuracy of 10 micro-arcsec, Gaia will provide accurate parallaxes and proper motions for a billion stars. Although the Gaia/RVS spectrometer will also provide radial velocities and elemental abundances for millions of stars, they are limited to stars brighter than 15-16 mag, about 2-3 mag, shallower than achievable with the LAMOST. Radial velocities and basic stellar parameters (effective temperatures, surface gravities and metallicities) of millions of Galactic stars derivable from LAMOST spectra, together with accurate distances and tangential velocities yielded by Gaia will allow one to study the stellar populations, kinematics and the star formation and chemical enrichment history of a significant volume of the Milky Way in unprecedented detail.

\section{Survey design}

The LSS-GAC will survey a contiguous sky area of $\sim 3,400$ sq.deg., covering Galactic longitudes $150 \leqslant l \leqslant 210^{\circ}$ and latitudes $|b| \leqslant 30^{\circ}$ (Fig. 1 ), and obtain $\lambda \lambda 3800-9000$ low resolution $(R \sim 1,800)$ spectra for a statistically complete sample of $\gtrsim 3 \mathrm{M}$ stars of all colors, uniformly and randomly selected from $(r, g-r)$ and $(r, r-i)$ Hess diagrams and in (RA, Dec) spatial distribution, obtained from the XSTPS-GAC, a CCD imaging photometric survey of $\sim 5,400$ sq.deg. using the Xuyi 1.04/1.20 m Schmidt Telescope. In order to make efficient use of observing time of different qualities and avoid fiber crosstalk, three categories of spectroscopic plates are designated, Bright, Medium-bright and Faint, targeting sources of brightness $14.0<r \lesssim m_{1}, m_{1} \lesssim r \lesssim m_{2}$ and $m_{2} \lesssim r \leqslant 18.5$, respectively. The border magnitude separating $\mathrm{B}$ and $\mathrm{M}$ plates, $m_{1}$, and that separating $\mathrm{M}$ and $\mathrm{F}$ plates, $m_{2}$, have values about 16.3 and 17.8 , respectively, but the exact numbers vary slightly from field to field, owing to the varying source density, stellar population and interstellar extinction. In general, $m_{1}$ and $m_{2}$ decrease towards the Galactic plane. Variable $m_{1}$ and $m_{2}$ are necessary in order to avoid discontinuities in the magnitude distributions of stars targeted by B, M and F plates, c.f. $\S 3$ for detail. Also note that during the early stage of the pilot survey, October 2011 - November 2011, the faint limit of $\mathrm{F}$ plates was set at 19.0 instead of 18.5.

The 4,000 fibers of LAMOST are distributed in a FoV of 20 sq.deg., yielding a fiber density of 200 per sq.deg. On average, $\sim 1,000$ stars will be surveyed per sq.deg. outside the thin disk $\left(|b|>3.5^{\circ}\right)$, implying 5 plates (allocated as $2 \mathrm{~B}, 2 \mathrm{M}$ and $1 \mathrm{~F}$ ) per pointing position. Within $|b| \leqslant 3.5^{\circ}$, the sampling is doubled. The LAMOST FoV however cannot be arbitrarily arranged, since for the active optics to work in order to bring individual segments of the primary and corrector mirrors into focus, there has to be a star brighter than $\sim 8 \mathrm{mag}$ at the field center. Given this constraint and considering that the LAMOST has a circular FoV, field centers are carefully chosen, yielding overlapping FoV's, such that the uniformity of spatial sampling over the whole survey area is maximized. The optimization is carried out for B, M and F plates separately. In total, 1,250 pointings are planned, including $500 \mathrm{~B}, 500 \mathrm{M}$ and $250 \mathrm{~F}$. 


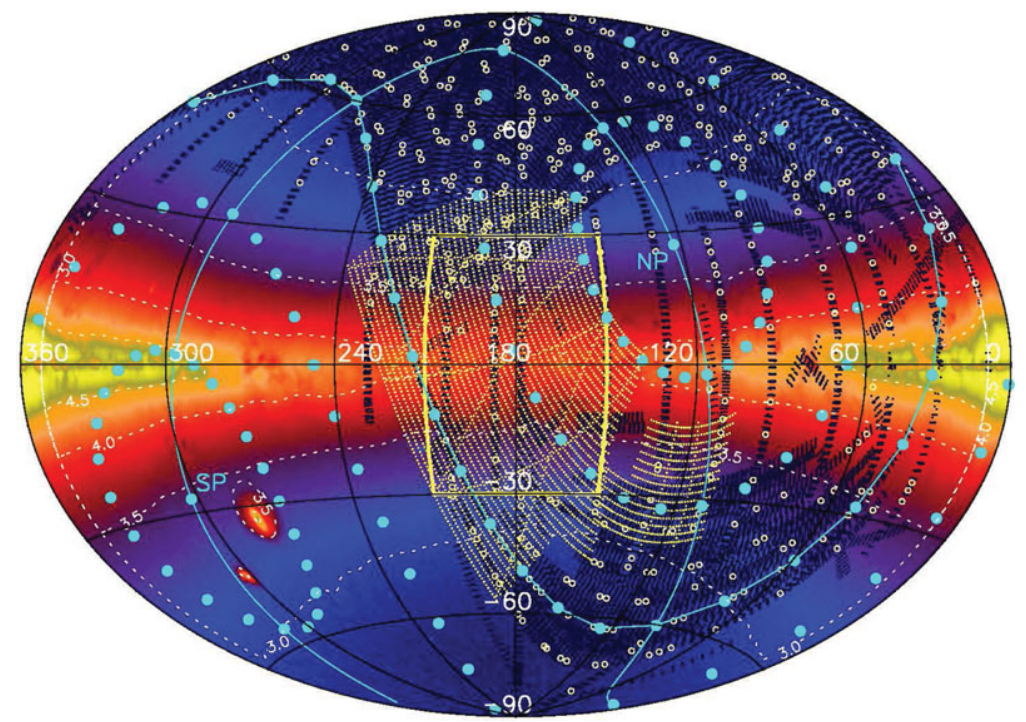

Figure 1. Footprints of the LSS-GAC (the central yellow bucket box) and XSTPS-GAC (the central yellow shaded area) in Galactic coordinates centered on the Galactic anti-center $\left(l=180^{\circ}, b=0^{\circ}\right)$. The background pseudo-color image shows the stellar number densities per squared degree from the 2MASS survey (Skrutskie et al. 2006), overlaid with contours of constant logarithmic number densities (the white dotted lines). The black shaded areas delineate footprints of the SDSS and SDSS-II imaging surveys. White open circles denotes SDSS/SEGUE spectroscopic plates. The cyan lines and dots delineate the equatorial coordinate system, with the north and south celestial poles marked.

In the overlapping areas of adjacent FoV's, fibers are assigned to stars regardless whether they have been assigned a fiber or not in the adjacent plates. As a result, $\sim 23 \%$ of all selected targets will actually be targeted twice, opening up the possibility of time-domain spectroscopy for, e.g. identifications of spectroscopic binaries.

Finally, observing time under bright lunar conditions are used to target very bright stars of magnitudes between $\sim 9$ and 14. They form an excellent sample of local stars to probe the solar neighborhood. The VB plates are implemented and scheduled separately.

\section{XSTPS-GAC and target selection}

To provide input catalogs for the LSS-GAC, XSTPS-GAC - the Xuyi Schmidt Telescope Photometric Survey of the Galactic Anti-center was initiated in the fall of 2009 and completed in the spring of 2011. The survey was carried out in SDSS $g_{-}, r$ - and $i$-bands with the Xuyi 1.04/1.20 m Schmidt Telescope equipped with a $4 \mathrm{k} \times 4 \mathrm{k}$ CCD camera, operated by the Near Earth Objects Research Group of the Purple Mountain Observatory. The CCD offers a FoV of $1.94^{\circ} \times 1.94^{\circ}$, with a pixel scale of 1.705 arcsec. The survey covers an area of $\sim 5,400$ sq.deg., from RA $\sim 3$ to $9^{\text {h }}$ and Dec $\sim-10$ to $+60^{\circ}$, plus an extension of $\sim 900$ sq.deg. to the M 31/M 33 area. Including the bridging fields connecting the two areas, the total survey area is close to 7,000 sq.deg (Fig. 1).

The survey was carried out under good observing conditions (but not necessarily photometric) in grey and dark nights. In a given night, fields of two adjacent stripes of constant declinations were observed in turn, stepping the field center in RA by half the FoV $\left(0.95^{\circ}\right.$, i.e. $\sim 50 \%$ overlap). The stepping in Dec between the stripes was set at $1.9^{\circ}$, yielding an overlap of $\sim 2$ arcmin between two adjacent stripes. With an exposure 

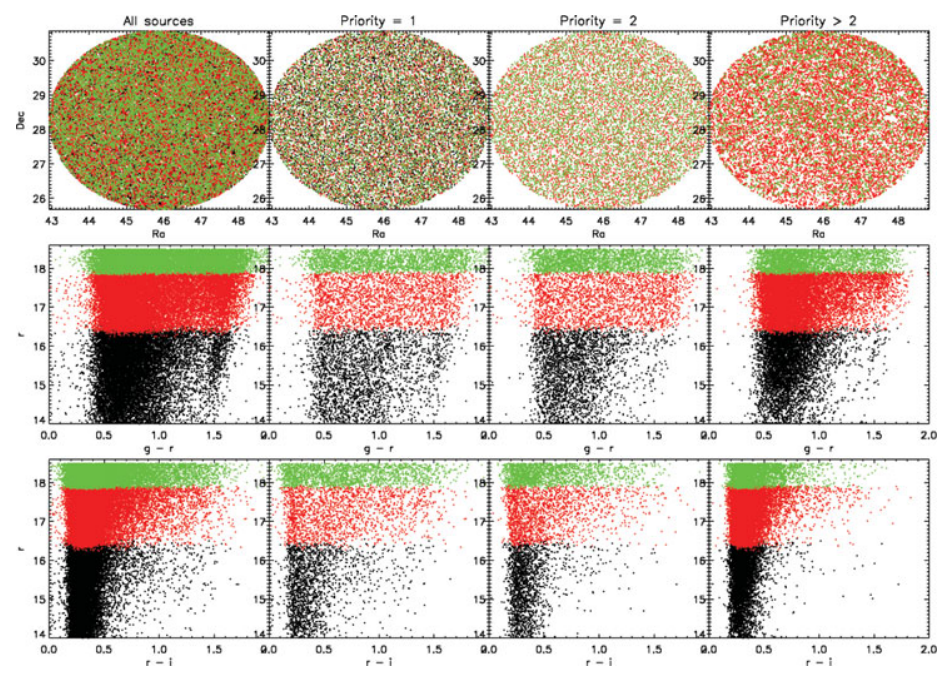

Figure 2. From top to bottom, (RA, Dec) spatial distribution and $(r, g-r)$ and $(r, r-i)$ Hess diagrams for all stars (first column), stars of first (second column), second (third column) and lower (fourth column) priorities in the clean sample, for a field around $\mathrm{RA}=3^{\mathrm{h}}$ and $\mathrm{Dec}=28^{\circ}$.

time of $90 \mathrm{sec}$ and a dual-channel readout time of $43.2 \mathrm{sec}$, the moving of telescope pointing direction as the observation proceeded was minimized, creating maximum uniformity amongst the fields. To facilitate tying all the fields to a common flux level ("ubercal"), "Z-stripe" fields that straddled the borders of adjacent "normal" stripes were added. The images were flat-fielded using "super-sky flat fields" generated from the target images themselves, after clipping all visible stars. Astrometric calibration was carried out using the PPMXL catalog (Roeser et al. 2010). Aperture and PSF photometric measurements were made using a modified DAOPHOT-based pipeline developed by the BATC group of National Astronomical Observatories of Chinese Academy of Sciences (NAOC), and then globally calibrated against the SDSS DR8 using overlapping sky areas. In total, the XSTPS-GAC archives approximately $100 \mathrm{M}$ stars down to a limiting magnitude of $\sim 19$ $(10 \sigma)$. The astrometric accuracy is about $0.1 \mathrm{arcsec}$, and a global photometric accuracy of $\sim 2 \%$ has been achieved.

The LSS-GAC targets are selected from the XSTPS-GAC photometric catalogs. The philosophy is to adopt a simple yet non-trivial algorithm, as uniform as possible over the whole survey area, such that whatever objects (e.g. white-dwarf-main-sequence binaries, extremely metal-poor or hyper-velocity stars) are revealed by the spectroscopic observations, they can be studied in a statistically meaningful way in terms of the underlying stellar populations (at least for the survey volume), after various selection effects introduced by the input catalog, those imposed by the constraints of instrument and observation (e.g. the positioning of field center, the holes in focal plane produced by the guiding CCDs and the central Shack Hartmann Sensor, allocations of fibers, spectral $\mathrm{S} / \mathrm{N}$, etc) have been properly taken into account. Meanwhile in order to increase the discovery space, rare objects of extreme colors (e.g. white dwarfs, red giants) should be preferentially targeted (Fig. 2).

The XSTPS-GAC photometric catalogs are first used to generate a clean sample, with the requirements that: 1) The stars are detected in at least two bands, including $r$-band, and have $14.0<r \leqslant 18.5 ; 2$ ) The star positions measured in different bands agree within 0.5 arcsec; 3$)$ They are not flagged as galaxies or star-pairs in either $r$ - or $i$-band; 4 ) 
They have no neighbors within 5 arcsec that are brighter than $m+1$ mag, where $m$ is the magnitude of star concerned, and are not neighbors of extremely bright stars.

To select targets, we first need to estimate values of $m_{1}$ and $m_{2}$, the border magnitudes between B, M and F plates (c.f. $\S 2$ for detail). For each survey area in a (RA, Dec) box of one degree wide, stars of extremely blue colors, $g-r$ or $r-i \leqslant-0.5$, and of extremely red colors, $g-r$ or $r-i>2.5$, are first selected. Stars in the remaining color space are then selected using a Monte Carlo method. First two random numbers, $r$ in the range $(14,18.5)$ and $c$ in $(-1,5)$, are generated. If $-1<c \leqslant 2$, then the $(r, g-r)$ Hess diagram is used, assuming $g-r=c+0.5$, otherwise the $(r, r-i)$ Hess diagram is used instead, assuming $r-i=c-2.5$. For each $(r, g-r)$ or $(r, r-i)$ set, if there are stars on the Hess diagram in a box centered on the simulated set of color-magnitude and of length 0.2 in magnitude and 0.3 in color, then the star of color-magnitude values closest to the simulated set is chosen and removed from the pool. If not, the process is repeated until a total of 1,000 stars, including those of extreme colors, per sq.deg. are selected. The stars are then sorted in magnitude from bright to faint. Then $m_{1}$, the border magnitude separating B and $\mathrm{M}$ plates is set to the faint end magnitude of the first $40 \%$ sources, and $m_{2}$ separating $\mathrm{M}$ and $\mathrm{F}$ plates is set to the faint end magnitude of the first $80 \%$ sources.

The above procedure applies to the survey area of $|b|>3.5^{\circ}$, where the LSS-GAC plans to sample 1,000 stars per sq.deg. For $|b| \leqslant 3.5^{\circ}$, the target density is doubled and 2,000 stars per sq.deg. are selected. In addition, in this case the selections are carried out in $(l, b)$ instead of $(\mathrm{RA}, \mathrm{Dec})$.

Once the values of $m_{1}$ and $m_{2}$ are set, stars of $\mathrm{B}, \mathrm{M}$ and $\mathrm{F}$ plates are selected, in batches of 200 stars per sq.deg. For each category, up to 10 batches can be selected pending on the availability of sources. The first batch of stars are assigned the highest observational priority, the second one priority lower, and so forth. In selecting each batch of stars, if a star of a given coordinate set, (RA, Dec) or $(l, b)$, is selected, then stars in its vicinity of 2 arcmin are removed from the pool. Those stars are however put back to the pool when selecting the next batch of stars.

For each selected field center (c.f. §2) and plate category (B, M or F), fibers are assigned to stars selected above according to their priorities by running through SSS, the LAMOST Survey Strategy System, assuming a nominal observing date and time. Fibers targeting blank sky for the measurements of sky background, typically 320 per plate, are also allocated. Excluding dead fibers $(\sim 100)$, generally $\sim 3,500$ fibers are available for the LSS-GAC targets per plate. The SSS has to be rerun near the actual observing date, to allow for the time-dependence of transformation between the equatorial and focal plane coordinate systems and to ensure enough guiding stars are accessible. The changes of stars that eventually get allocated a fiber and observed are generally small, typically on the level of a few tens or less.

The above survey strategy and target selection algorithm are tested using mock catalogs generated from the Besançon Galactic model (Robin et al. 2003). As an example, for a $1^{\circ}$ wide stripe of $56.7 \mathrm{sq} . \mathrm{deg}$. centered at $l=180^{\circ}$ and stretching from $b=-30$ to $+30^{\circ}$, the model yields 396,075 stars of $14<r \leqslant 18.5 \mathrm{mag}$. Amongst them, 0.018, 2.3 and $2.1 \%$ are $\mathrm{M}, \mathrm{K}$ and $\mathrm{G}$ subgiants/giants, respectively, and 1.9, 24, 39, 24 and $4.3 \%$ are respectively $\mathrm{A}, \mathrm{F}, \mathrm{G}, \mathrm{K}$ and $\mathrm{M}$ dwarfs. Running this mock catalog through the above target selection algorithm, one finds that in total 64,029 of all stars, 99, 47 and $27 \%$ of all M, K and G subgiants/giants, 39, 14, 8, 19 and $55 \%$ of all A, F, G, K and $\mathrm{M}$ dwarfs, will be selected and targeted by the LSS-GAC, respectively. Fig. 3 shows the spatial distributions of various types of star targeted in the $(X, Z)$ plane of Galactic radius and height. 

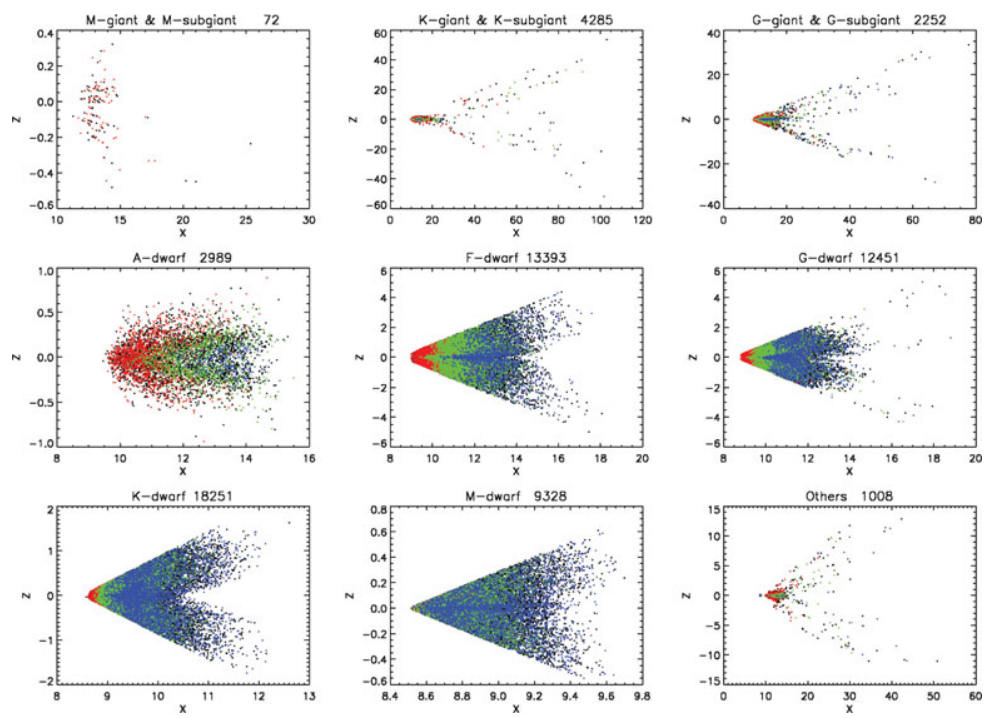

Figure 3. Spatial distributions of the simulated LSS-GAC targets in the $(X, Z)$ plane of Galactic radius and height for a one-degree wide stripe of $|b| \leqslant 30^{\circ}$ centered at $l=180^{\circ}$. The numbers of different types of star targeted in this stripe are labeled. Red, green and blue dots represent sources of B, M and F plates, respectively (c.f. Fig. 2).

\section{Survey status}

After the completion of construction in June 2009, LAMOST went through a two-year period of commissioning and performance characterization and optimization. The problem of fiber-positioning was solved using an innovative technique. The 4,000 fibers are divided into 160 groups. For each group, the 25 adjacent fibers are utilized to target 25 bright stars, with predefined offsets following a given pattern. The measured throughput ratios of stellar and sky light of the 25 fibers are then used to estimate the average fiber positioning error of this group of fibers in the focal plane. With this method, the distribution of fiber positioning errors can be mapped out with a single exposure, even under non-photometric conditions (Yuan et al., in preparation). Slit masks of $2 / 3$ the fiber diameter were also introduced, increasing the spectral resolution from $\sim 1,200$ to 1,800 , comparable to that of SDSS, at the expenses of $20 \%$ loss of light.

Following the commissioning period, a pilot survey of one year was initiated in the fall of 2011, to further test and optimize the survey strategy. Constant monitoring of the LAMOST performance shows that for large scale surveys, a limiting magnitude of 17.8 is probably realistic. Under exceptional conditions (a few nights per months), a depth of 18.5 mag can be reached for limited number of fields.

The LAMOST formal surveys were initiated in the fall of 2012. Hitherto, within the LSS-GAC footprint, $\sim 0.15$ and $0.25 \mathrm{M}$ spectra of $\mathrm{S} / \mathrm{N}(\lambda 7450) \geqslant 10$ per pixel $(\sim 1.7 \AA)$ have been collected during the periods of pilot and formal surveys, respectively. In addition, $\sim 0.4 \mathrm{M}$ spectra from the VB plates (some outside the LSS-GAC footprint) have been obtained. The spatial distributions of those sources are shown in Fig. 4.

\section{Data reduction}

The LAMOST 2D, 1D and stellar parameter pipelines have been developed at the LAMOST Operation and Development Center (Luo et al., this volume). There are 

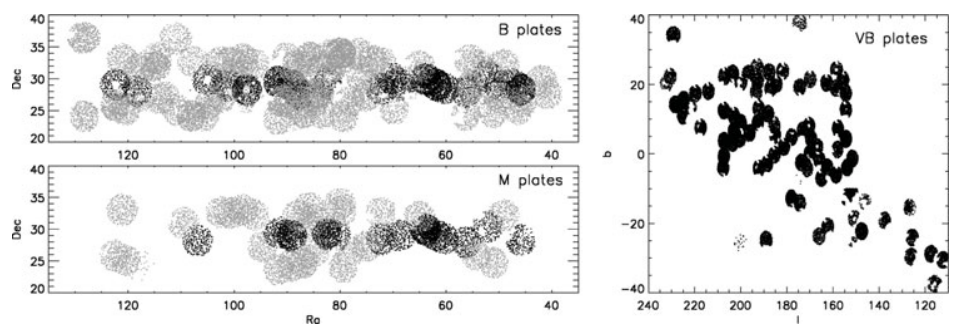

Figure 4. Spatial distributions of targets from B (top left), $M$ (bottom left) and VB plates (right). By March 8, 2013, in total 257,317, 115,054, 25,480 and 393,791 spectra of $\mathrm{S} / \mathrm{N}(7450 \AA) \geqslant 10$ per pixel have been collected from B, M, F and VB plates, respectively. For $\mathrm{B}$ and $\mathrm{M}$ plates, black and grey dots show respectively data collected during the pilot and formal surveys. Only one-in-ten stars are shown.

however issues specific to the LSS-GAC, and a set of pipelines, LSP3, tailored to the need of LSS-GAC have been developed at Peking University (PKU).

Owing to high dust reddening in the disk $\left(E_{B-V} \sim 0.3\right.$ towards Galactic anti-center sightlines) flux calibration standards, such as F turnoff stars adopted by the SDSS, cannot be reliably identified and selected from the photometric colors alone. On the other hand, stars of all types and colors are targeted by the LSS-GAC. LSS-GAC spectra are first processed using nominal spectral response curves (SRCs). The initial stellar parameters are then derived using the LSP3. Based on the results, $5-10 \mathrm{~F}$ stars $\left[5,500<T_{\text {eff }}<\right.$ $7,000 \mathrm{~K}$ and $\log g\left(\mathrm{~cm} \mathrm{~s}^{-2}\right)>3.0$ ] per spectrograph are selected as the flux calibration standards. Extinctions towards those stars are deduced by comparing the photometric colors to those calculated from the Kurucz low-resolution model spectra (Castelli \& Kurucz 2004) of the same stellar parameters. The SRCs are then derived by comparing the observed spectra with the synthetic spectra of Munari et al. (2005), interpolated to the corresponding stellar parameters and degraded to the LAMOST spectral resolution, and then reddened assuming the reddening derived above. The Fitzpatrick (1999) extinction law of $R=3.1$ is adopted. The spectra are then reprocessed using the newly deduced SRCs and values of stellar parameters are updated. The above process is iterated (twice is sufficient) to yield the final flux-calibrated spectra.

As a test of the accuracy of our flux-calibration procedure, colors are calculated from the calibrated spectra and compared to the photometric values. For spectra of $\mathrm{S} / \mathrm{N}(\lambda 4650)>30$ per pixel $(\sim 1 \AA)$, we find $\Delta(g-r)=0.02 \pm 0.06$ mag on average. For $(r-i)$, the spectra yield colors about $0.06 \pm 0.04$ mag bluer than the photometric values, owing to the fact that the LSP3 has opted to not to correct for the telluric absorption bands, most notably in the $i$-band. Comparisons of the LAMOST and SDSS DR7 spectra of common objects show that for high extinction regions, the LSP3 yields more realistic spectral energy distributions (SEDs) than the SDSS. Finally, comparisons of the spectra obtained at different epochs indicate a calibration accuracy of better than $8 \%$ for the wavelength range $\lambda \lambda 4000-9000$. The SRCs are found to vary with time, by as much as $20 \%$ in a given night and even larger for different nights. The reasons are unclear. For the moment, it seems essential to derive SRCs for each observed plate.

The LSP3 derives stellar parameters by matching the observed spectra to the empirical spectral library MILES (Sánchez-Blázquez et al. 2006). Compared to the ELODIE library (Prugniel \& Soubiran 2001), the MILES library has the advantage that they are obtained with a spectral resolution $(2.3 \AA)$, comparable to that of LAMOST, and carefully fluxcalibrated. Both libraries have similar numbers of stars and parameter space coverage, 
with the parameters determined from high resolution spectroscopy. For radial velocity determinations, the ELODIE 3.1 library is used.

To obtain the initial values of radial velocity $V_{\mathrm{r}}$ and stellar parameters $T_{\text {eff }}, \log g$ and $[\mathrm{Fe} / \mathrm{H}]$ of a target spectrum, we first normalize the spectrum in a way similar to the SSPP (Lee et al. 2008), using a ninth and fourth order polynomial for the blue$(\lambda \lambda 3800-5800)$ and red-arm $(\lambda \lambda 6100-9000)$ spectra, respectively, and compare the results to the normalized MILES spectra. The biweight means and standard deviations of stellar parameters of the best 20 matches are then adopted as the initial parameters of the target spectrum. The initial $V_{\mathrm{r}}$ is then obtained by cross-correlating with the best match.

For further iterations, we first fix $V_{\mathrm{r}}$ and select a $3 \mathrm{D}$ box in the $T_{\text {eff }}, \log g$ and $[\mathrm{Fe} / \mathrm{H}]$ parameter space centered at the initial values. The sizes of box are set to 3 times the standard deviations of initial values or values $0.2 \times T_{\text {eff }}$ in $T_{\text {eff }}, 1.0$ dex in $\log g$ and 1.0 dex in $[\mathrm{Fe} / \mathrm{H}]$, whichever is bigger for each of three quantities. The observed target spectrum, un-normalized, is then matched with the MILES spectra, again un-normalized, in the box. To account for the possible effects of interstellar extinction and uncertainties in flux-calibration, a third order polynomial is allowed to scale the SEDs of MILES spectra to that of the target. The optimization is carried out separately for two wavelength ranges, $\lambda \lambda 4000-5500$ and $\lambda \lambda 6100-6800$, using both the minimum $\chi^{2}$ and maximum crosscorrelation techniques. Strong city light emission lines and night sky emission lines, e.g. the $\mathrm{Hg}$ I $\lambda 4358$ and [O I] $\lambda \lambda 6300,6363$, are masked out. With the newly determined stellar parameters, $V_{\mathrm{r}}$ is then redetermined using the ELODIE library. The process is repeated until $V_{\mathrm{r}}$ varies less than $3.0 \mathrm{~km} \mathrm{~s}^{-1}$ in two consecutive iterations. In most cases, the results converge in one or two iterations. The biweight means of stellar parameters of the four best templates in the final iteration are then adopted as the final values of the target spectrum. Currently, only results from the blue wavelength range are used.

Comparisons of multi-epoch observations, and with the results of RAVE (Steinmetz et al. 2006) and SDSS show that for F, G and K stars, the LSP3 has achieved an accuracy of 8 and $5 \mathrm{~km} \mathrm{~s}^{-1}$ for $\mathrm{S} / \mathrm{N}(\lambda 4650)>10$ and 20, respectively. For hotter stars, the uncertainties are $\sim 20$ and $7 \mathrm{~km} \mathrm{~s}^{-1}$ for $\mathrm{S} / \mathrm{N}(\lambda 4650) \gtrsim 10$ and 20 , respectively. For stellar parameters, the uncertainties are about $110 \mathrm{~K}, 0.15 \mathrm{dex}$ and $0.15 \mathrm{dex}$ for $T_{\text {eff }}, \log g$ and $[\mathrm{Fe} / \mathrm{H}]$, respectively. The stellar parameters yielded by LSP3 agree well with those given by ULySS (Koleva et al. 2009; Wu et al. 2012), a pipeline based on the ELODIE library. Fig. 5 compares the LSP3 radial velocities and stellar parameters determined from SDSS spectra with those of SDSS DR7 for member stars of two open clusters and two globular clusters. Some artifacts are clearly visible in the distributions of LSP3 parameters, owing to incomplete or sparse parameter coverage of the MILES stars, in particular at low metallicities. Efforts are underway to fill the gaps in parameter space of the MILES library by obtaining additional spectra using the NAOC $2.16 \mathrm{~m}$ telescope.

Fig. 6 shows distributions of radial velocities and stellar parameters determined for 197,821 spectra of $\mathrm{S} / \mathrm{N}(\lambda 4650)>10$ per pixel obtained with $\mathrm{B}, \mathrm{M}$ and $\mathrm{F}$ plates, and for 393,791 spectra obtained with VB plates. Artifacts apparent in the SDSS DR7 and DR9 releases, e.g. two false branches of stars in the $T_{\text {eff }}-\log g$ HR diagram, one near the turnoff stars $\left(T_{\text {eff }} \sim 6,200 \mathrm{~K}\right)$ and another at low temperatures $\left(4,000 \lesssim T_{\text {eff }} \lesssim 4,800 \mathrm{~K}\right)$, are no longer present. The LSP3 also seems to yield stellar parameters in better agreement with the theoretical isochrones.

\section{Examples of application}

Although the LSS-GAC survey and data reduction are still in early stage, nearly one million spectra and resultant radial velocities, stellar parameters are already available 

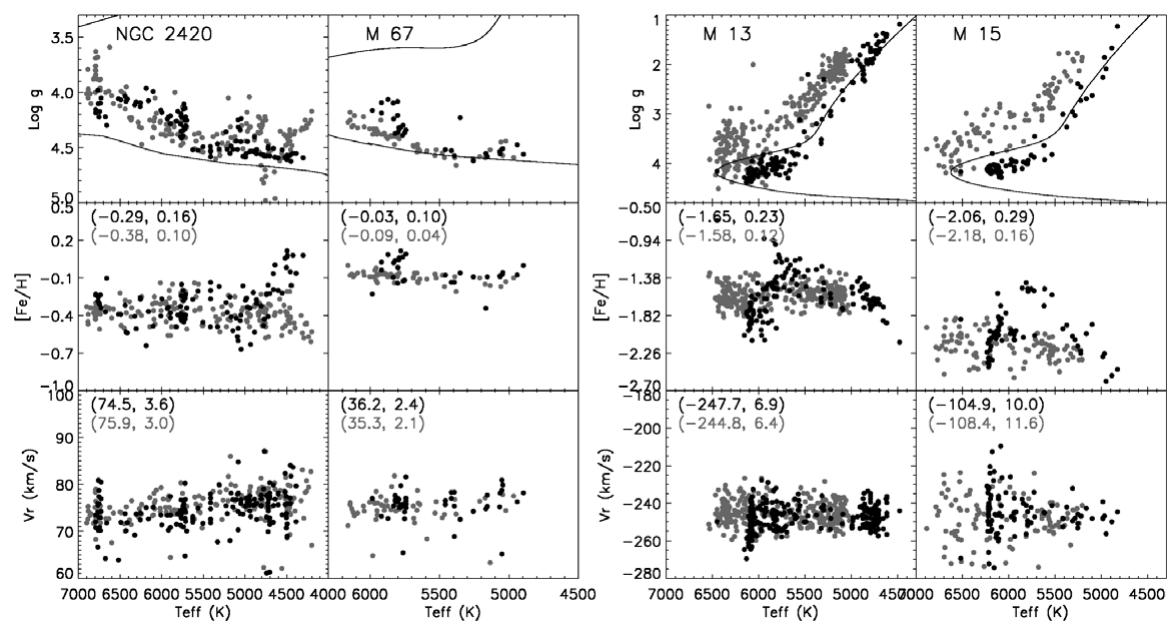

Figure 5. Comparisons of LSP3 (black dots) and SDSS DR7 (grey dots) stellar parameters for the open clusters NGC 2420 and M 67, and globular clusters M 13 and M 15. For M 67 and M 15, a constant value of +40 and $-140 \mathrm{~km} \mathrm{~s}^{-1}$ has been added to the measured values, respectively. Also overplotted in the top panels are isochrones of $([\mathrm{Fe} / \mathrm{H}],[\alpha / \mathrm{Fe}]$, age $)=(-0.3,0,1 \mathrm{Gyr})$ for NGC 2420, $(0,0,2.5 \mathrm{Gyr})$ for M $67,(-1.5,0.4,11.5 \mathrm{Gyr})$ for M 13 and $(-2.0,0.4,12 \mathrm{Gyr})$ for M 15. The clustering of LSP3 points at high temperatures are due to the scarceness of MILES stars at those temperatures, particularly at low metallicities.
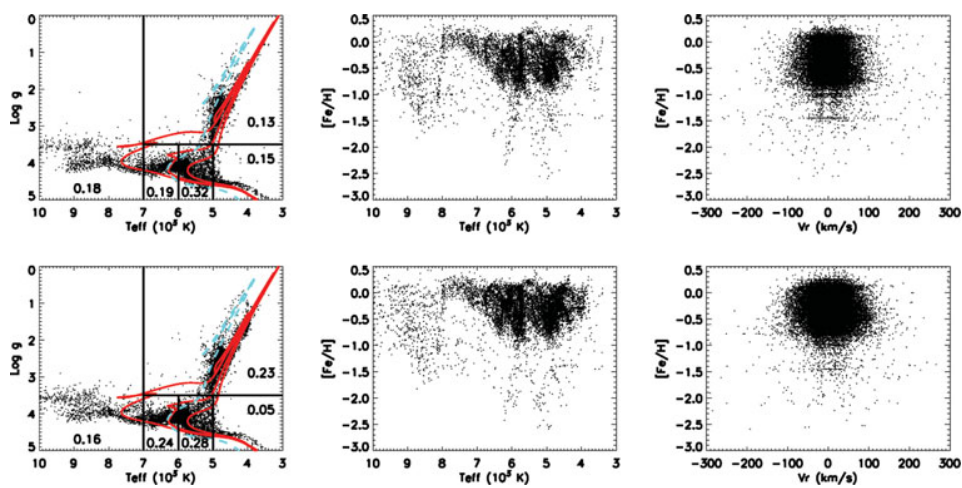

Figure 6. Radial velocities and stellar parameters determined from 197,821 spectra of B, M and F plates (top panels) and 393,791 spectra of VB plates (bottom panels) of $\mathrm{S} / \mathrm{N}(\lambda 4650)>10$ per pixel. Only one-in-ten stars are shown. In the left two panels, the cyan dashed line shows the isochrone of metallicity $[\mathrm{Fe} / \mathrm{H}]=-1$ and age $10 \mathrm{Gyr}$, while the three red lines, from left to right, show the isochrones of $[\mathrm{Fe} / \mathrm{H}]=0$ and ages 1,3 and $10 \mathrm{Gyr}$, respectively. The $T_{\text {eff }}-\log g$ plane is divided into different regions. The fractions of stars of individual regions are labeled.

for scientific exploitation. Taking advantage of the large number of spectra offered by the SDSS/SEGUE and using an innovative technique of paring stars of high extinctions with those of very low or nil extinctions but otherwise of identical stellar parameters, Yuan \& Liu (2012) report the detections of diffuse interstellar bands towards thousands of sightlines of Galactic stars. The same technique has been utilized to derive the extinction coefficients and study the extinction law towards thousands of sightlines (Yuan, Liu \& Xiang 2013). Applying this technique to the LSS-GAC data, Yuan et al. (this volume) has produced 3D extinction maps of the outer parts of Galactic disk. In spite of the incomplete sky coverage, distinct features, such as the Perseus and Outer Arms, and effects of warps of the outer disk, are clearly visible in the deduced extinction maps. In the work, once 

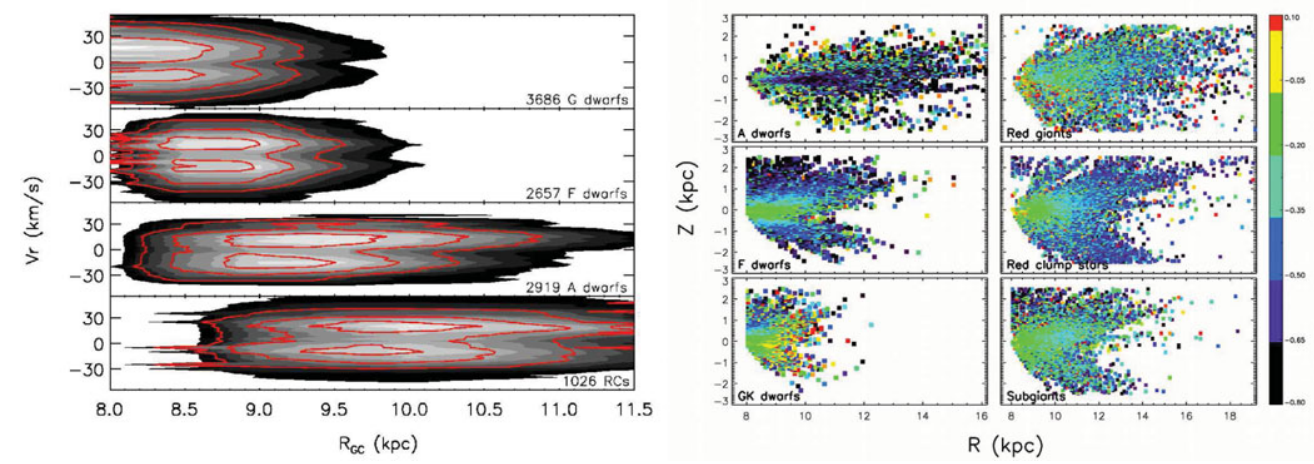

Figure 7. Left: Bifurcation of radial velocities of disk G, F and A dwarfs and RCs of heights $|Z|<0.1 \mathrm{kpc}$ in the direction of Galactic anti-center $\left(178<l<182^{\circ}\right)$. Right: Metallicity distributions traced by different types of star.

the extinction towards the sightline of a given star has been determined by combining the XSTPS-GAC and 2MASS photometry, given the $T_{\text {eff }}, \log g$ and $[\mathrm{Fe} / \mathrm{H}]$ parameters yielded by LSP3, the star's distance is then estimated by finding the closest model in the Dartmouth Stellar Evolution Database (Dotter et al. 2008) except for red clump stars (RCs). Reliability and accuracy of the algorithm are tested using multiple observations, star clusters and the Hipparcos distances of stars in the MILES and ELODIE libraries. We find that the algorithm yields distances accurate to $\sim 15$ and $30 \%$ for dwarfs and giants, respectively, when the spectra have $\mathrm{S} / \mathrm{N}(\lambda 4650) \geqslant 15$. However, we also find that the distances thus derived are systematically smaller than the Hipparcos values by respectively $\sim 6$ and $12 \%$ for dwarfs and giants. RCs are good standard candles and easily identifiable in the $T_{\text {eff }}-\log g \mathrm{HR}$ diagram, falling in the region of $4,500<T_{\text {eff }}<5,200 \mathrm{~K}$ and $2<\log g\left(\mathrm{~cm} \mathrm{~s}^{-2}\right)<3$. Their distances are calculated assuming absolute magnitudes $M_{i}=0.189$ and $M_{K s}=-1.567$ (Gronewegen 2008), with an estimated accuracy of $\sim 10 \%$. Currently, a Bayesian approach as proposed by Burnett \& Binney (2010) is being considered for implementation in order to determine stellar distances, ages, masses and metallicities simultaneously.

Liu et al. (2012) find that the radial velocities of a sample of 697 RCs observed with the MMT $6.5 \mathrm{~m}$ telescope show a bifurcation distribution split by $30 \mathrm{~km} \mathrm{~s}^{-1}$, peaking at Galactic radius of $\sim 10 \mathrm{kpc}$, in coincidence with the location of the Perseus arm, and interpret the result as evidence of the presence of the outer Lindblad resonance of the Galactic bar, although the possibility of the corotation resonance of the spiral arms can not be ruled out. The left panels of Fig. 7 show the radial velocity distributions of thousands of disk G, F, A dwarfs and RCs from the LSS-GAC survey in the direction of Galactic anti-center. Bifurcation is clearly seen in all cases, peaking at different radii, from the solar neighborhood to $\sim 11 \mathrm{kpc}$, in line with the expected survey depths of individual types of tracer of concern here. In fact, a bifurcation distribution is seen in all directions, from longitudes 150 to $210^{\circ}$ and of Galactic heights $|Z| \leqslant 0.8 \mathrm{kpc}$, casting doubt upon a resonant interpretation.

The right panels of Fig. 7 show metallicity distributions traced by different types of star. While the data including distances need to be carefully checked, they have already shown some interesting trends, e.g. significant vertical but marginal radial gradients. It is also interesting to note that RCs seem to indicate a clear radial gradient beyond $10 \mathrm{kpc}$. 
In conclusion, the LSS-GAC is well under way and poses to make major contributions to our understanding of the stellar populations, kinematics, and the chemical enrichment and star formation history of the Milky Way.

\section{References}

Ahn, C. P., et al. 2012, ApJS, 203, 21

Burnett, B., \& Binney, J. 2010, MNRAS, 407, 339

Castelli, F., \& Kurucz, R. L. 2004, astro-ph, 0405087

Cui, X.-Q., Zhao, Y.-H., Chu, Y.-Q, et al. 2012, RAA, 12, 1197

Dotter, A., Chaboyer, B., Jevremović, D., et al. 2008, ApJS, 178, 89

Eisenstein, D. K., et al. 2011, AJ, 142, 72

Fitzpatrick, E. L. 1999, PASP, 111, 63

Groenewegen, M. A. T. 2008, A\&A, 488, 935

Koleva, M., Prugniel, P., Bouchard, A., \& Wu, Y. 2009, A\&A, 501, 1269

Lee, Y. S., et al. 2008, AJ, 136, 2022

Liu, C., Xue, X.-X., Fang, M., et al. 2012, ApJL, 753, L24

Mayer, L., Governato, F., Kaufmann, T. 2008, ASL, 1, 7

Munari, U., Sordo, R., Castelli, F., \& Zwitter, T. 2005, A\& A, 442, 1127

Perryman, M. A. C., de Boer, K. S., Gilmore, G., et al. 2001, A\&SA, 369, 339

Prugniel, P., \& Soubiran, C. 2001, A\&A, 369, 1048

Robin, A. C., Reylé, C., Derrière, S., Picaud S. 2003, A\&A, 409, 523

Roeser, S., Demleitner, M., \& Schilbach, E. 2010, AJ, 139, 2440

Sánchez-Blázquez, P., et al. 2006, MNRAS, 371, 703

Schlegel, D. J., Finkbeiner, D. P., Davis, M. 1998, ApJ, 500, 525

Skrutskie, M. F., et al. 2006, AJ, 131, 1163

Steinmetz, M., Zwitter, T., Siebert, A., et al. 2006, AJ, 132, 1645

Wu, Y., Luo, A.-L., Li, H.-N., et al. 2012, RAA, 11, 924

Yanny, B., Rockosi, C., Newberg, H. J., et al. 2009, AJ, 137, 4377

York, D. G., et al. 2000, AJ, 120, 1579

Yuan, H.-B. \& Liu, X.-W. 2012, MNRAS, 425, 1763

Yuan, H.-B., Liu, X.-W., \& Xiang, M.-S. 2013, MNRAS, 430, 2188

\section{Discussion}

Poul Erik Nissen: You mentioned the possibility to measure $[\alpha / \mathrm{Fe}]$ and $[\mathrm{C} / \mathrm{Fe}]$. Do you have any results on that already?

XiaOwei LiU: Not yet. The possibility is demonstrated by Marstellar et al. (2009, AJ, 138, 533) and Lee et al. (2011, AJ, 141, 90), although we believe the methods need careful calibration.

JAMES Binney: Are you delivering distances in parallel with the other parameters? Can you say something about the accuracy of your distances?

XIAOWEI LIU: Yes, extinctions and distances will be released along with the LSP3 parameters, and can be obtained from the author (XWL) upon request, subject to regulations of the LAMOST data policy. A brief discussion of the accuracy is presented in $\S 6$.

HANS-Günther LUDWIG: What is the data policy of the LSS-GAC survey? Will and if so when will the data be released to the community?

XIAOWEI LIU: LSS-GAC is parts of the LAMOST surveys and follows the same data policy. 